\title{
Écosystème
}

\section{DE LA DANSE AU MUSÉE AU MUSÉE DE LA DANSE}

\section{Eloïse Guénard}

Volume 2, numéro 1, 2019

Danse

URI : https://id.erudit.org/iderudit/1062561ar

DOI : https://doi.org/10.7202/1062561ar

Aller au sommaire du numéro

Éditeur(s)

La chambre blanche

ISSN

2562-3222 (numérique)

Découvrir la revue

Citer cet article

Guénard, E. (2019). DE LA DANSE AU MUSÉE AU MUSÉE DE LA DANSE.

Écosystème, 2(1), 35-42. https://doi.org/10.7202/1062561ar d'utilisation que vous pouvez consulter en ligne.

https://apropos.erudit.org/fr/usagers/politique-dutilisation/ 


\section{DE LA DANSE AU MUSÉE AU MUSÉE DE LA DANSE}

\section{Eloïse Guénard}

Depuis quelques années, le nombre d'invitations faites à la danse d'entrer au musée n'a cessé d'augmenter. Plusieurs musées ont créé des départements dédiés à la performance (en arts visuels et dans le champ des arts vivants), des aménagements spécifiques ont été conçus et quelques pièces chorégraphiques ont intégré des collections publiques ${ }^{29}$. Les conceptions et les formats engagés n'en demeurent pas moins hétérogènes, qu'ils répondent à une intention pédagogique, événementielle, patrimoniale ou esthétique (de l'atelier à l'exposition historique, de la visite dansée face aux oeuvres à la programmation chorégraphique indépendante).

Clin d'œil au titre de la revue, Écosystème, ce texte combine une approche esthétique et institutionnelle, avec pour toile de fond le dialogue historique de la danse et des arts visuels. Comment les musées ou les centres d'art, traditionnellement dévolus à des objets, prennent-ils en charge la présentation du corps vivant et pourquoi ? Que devient une chorégraphie dont les codes de représentation sont mis à l'épreuve du musée ?

\section{Une histoire dansée}

À considérer le musée comme un lieu de conservation, où s'écrit de surcroît l'histoire de l'art, le bénéfice réciproque de ce rapprochement se laisse aisément deviner. La danse y trouverait-elle un remède à sa nature éphémère ? Faut-il le rappeler, une chorégraphie, en tant qu'art du geste, ne laisse que de rares traces, sans appartenir non plus au régime allographique qui caractérise la musique ${ }^{30}$. L'urgence de la préservation de sa mémoire surgit violemment quand le milieu se voit frappé par le sida dans les années 1980, relancée dernièrement par la perte de grandes figures tutélaires de la danse contemporaine. Le musée, de son côté, continuerait sa mue pour accueillir les pratiques les plus contemporaines et se défaire du caractère de sanctuaire, voire de cimetière dont il a pu être incriminé $^{31}$. Néanmoins, cette ambition s'expose à différents paradoxes.

29 Parmi d'autres, citons les expositions et programmations : Dancing museums avec cinq musées européens (20152017); Le musée comme performance (Musée d'art contemporain de Serralves, Porto, 2017); Corps rebelles (Musée de la civilisation, Québec, 2016) ; Dancing around the bridge (Philadelphia Museum of Art, 2013) ; Some sweet day (MoMA, 2012) ; Move : Chorégraphing you (Hayward Gallery, Londres, 2010 ; Düsseldorf, 2011 ; Séoul, 2012) ; Danser sa vie (Centre Pompidou, Paris, 2011); Dance/Draw (ICA, Boston, 2011); The living Currency (Tate Modern, Londres, 2008), Une exposition chorégraphiée (La Ferme du Buisson, Noise-Le-Grand, 2008). En 2007, Aurélie Gandit chorégraphie des "visites dansées » au Musée des Beaux-Arts de Nancy. Mark Franko et André Lepecki dans l'édito du numéro Dance in the Museum notent également que le prix de la biennale du Whitney a été décernée à une chorégraphe, Sarah Michelson, pour sa pièce Devotion: The American Dancer et le Lion d'or de la Biennale de Venise 2013 à Tino Sehgal. La Ribot propose des «instants dansés » dans les musées dès les années 1990. En 2002, la Tate Modern inaugure une série de performance dans le Turbine Hall ; en 2009, le MoMA crée un département média et performance.

30 Nous renvoyons à Frédéric Pouillaude qui, à partir du constat de l'absence de production et de conservation, forge la notion de «désœuvrement» pour qualifier le régime spécifique de l'oeuvre en danse dans Le désoeuvrement chorégraphique, étude sur la notion d'oeuvre en danse, Paris, Vrin, 2009. 432 p.

31 Dès 1861, Théophile Thoré déclarait : «Les musées ne sont que des cimetières de l'art, des catacombes où l'on range dans une promiscuité tumulaires les restes de ce qui a vécu [...]», dans Théophile Thoré (alias William Bürger), Salon de 1861, dans Salons de W. Bürger, Paris, Renouard, 1870, vol. 1, p. 84-85. Théodor Adorno dira plus tard 
À partir des années 1990, la volonté de prendre en charge l'histoire de la danse et d'en créer une archive vivante, par exemple à travers des formes de reenactment, apparaît chez différents chorégraphes. Sans doute la réflexion patrimoniale dont le musée est investi s'inscrit-elle dans ce contexte. Le titre de plusieurs pièces en témoigne. 20 danseurs pour le $X X^{e}$ siècle de Boris Charmatz, donné entre autres au MoMA, à la Tate Gallery et au $\mathrm{Mac} / \mathrm{Val}$, déploie une histoire de la danse dans l'espace. Les interprètes disséminés dans les salles, couloirs et escaliers exécutent des morceaux choisis, d'Isadora Duncan à Vaslav Nijinsky, de Merce Cunningham à William Forsythe, de Pina Baush à Dominique Bagouet et jusqu'à Michaël Jackson. Avec le titre évocateur de Restrospective (Fondation Tapiès, Barcelone, 2012, Centre Pompidou et MoMA PS1, 2014, notamment), l'exposition chorégraphique de Xavier Le Roy emprunte une voie comparable par la temporalité qu'elle instaure. L'interférence entre arts du temps et de l'espace y apparaît explicitement. Une vingtaine d'interprètes s'approprient des extraits solo réalisés par Xavier Le Roy entre 1994 et 2010. Dans le numéro de Repères, cahier de danse, «Danse et/au musée $e^{32}$ », Scarlet Yu, qui a collaboré à la pièce, explique comment la chronologie prévalente dans les arts visuels est ici transposée en une action.

Figure 3: $\quad$ Xavier Le Roy, Retrospective, MoMA PS1, New York, 2014. Photo reproduite avec l'aimable autorisation de C Matthew Septimus et MoMA PS1

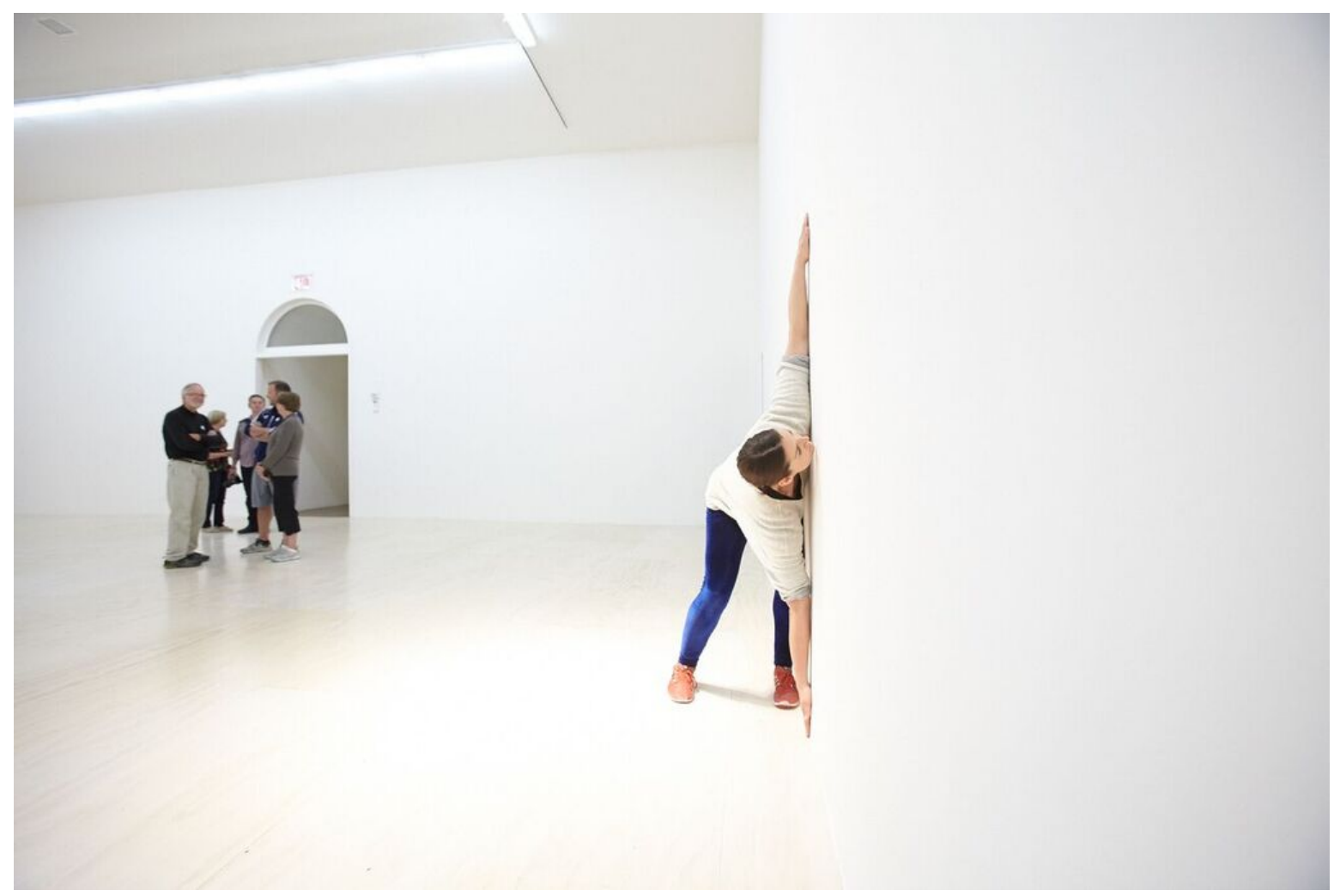

«Les musée sont les sépultures familiales des œuvres d'art » - citation par laquelle l'historien d'art américain Douglas Crimp ouvre son Museums' Ruins (1980).

32 «Exposer la danse au musée. Entretien avec Scarlet Yu », dans Repères, cahier de danse, «Danse et/au musée », Vitry sur-Seine, La Briqueterie, 2017, pp 5-9. 
L'institution muséale déplace-t-elle le dialogue déjà ancien de la danse avec les arts visuels sur le terrain historiographique ? Jérôme Bel s'y attelle dans le travail qu'il n'a cessé de mener sur le dispositif scénique, tout en s'appropriant certaines formes issues de l'histoire des arts visuels ${ }^{33}$. Dans son Catalogue raisonné, mis en ligne et composé de films, entretiens, monologues et extraits de $\operatorname{spectacles}^{34}$, il orchestre l'appareil critique de sa propre œuvre et son archivage. À la peur de la disparition et de l'incompréhension, se mêle une volonté de transmission, présente dès les pièces Véronique Doisneau (2004), Pitchet Klunchun and myself (2005) ou Cédric Andrieux (2009). Les danseurs livrent, par le récit de leur parcours, un aspect de l'histoire de la danse (le ballet classique, la danse traditionnelle thaïlandaise, la danse moderne et contemporaine). La neutralité affichée, que (seule) la littéralité des titres viendrait corroborer, fait plutôt du document et du témoignage une forme de représentation. Pour autant, régulièrement sollicité par des musées et biennales, il précise dans un entretien : «De ce que j'ai pu expérimenter jusqu'ici, j'ai constaté que ce qui était proposé, soit restait en dessous du théâtre, soit n'était pas assez pensé pour le musée », tandis qu'il affirme ne pas faire pour sa part d'expositions dansées ${ }^{35}$.

Dans ces exemples, le musée participe d'un ancrage historique et d'une réflexion sur le médium chorégraphique, entre immatérialité et volonté (ou pas) de faire trace, éphémère du geste et réactualisation, transitoire et transmission. Le rapport à la temporalité confronte, avec l'abolition du quatrième mur et de la scène, les conventions muséales et scéniques. La durée est étirée, le déroulement se libère d'une progression linéaire entre un début et une fin et l'espace se fragmente. L'abandon de la frontalité inhérente à la scène au profit d'un espace à 360 degrés fait des visiteurs, de leurs circulations et de leurs perceptions une composante de la pièce. In fine, l'histoire de la danse et la plasticité de la durée se font sujet, contexte et matériau.

\section{De l'expérimentation à l'événement}

Mais, la danse n'y perdrait-elle pas autant qu'elle y gagne?

Déjà dans le sillage du Black Mountain College puis du Judson Dance Theater aux ÉtatsUnis, les formats hors plateau ont constitué le levier d'une remise en cause de la narration et de la dramaturgie en danse. Mais, les tenants de la dance postmoderne n'ont eu de cesse qu'ils ne gagnent la rue avec le développement de pratiques in situ œuvrant à un rapprochement de l'art et du monde quotidien. Du côté des arts visuels, la sortie du musée a été autant le fer de lance d'une critique institutionnelle autant qu'une nécessité face à la frilosité des musées à intégrer recherches et transgressions.

\footnotetext{
33 Pratique qui avait pu être qualifiée de danse «plasticienne », «performative », « conceptuelle » ou encore de « non danse » (Dominique Frétard, « La fin annoncée de la non-dance », Le Monde, 6 mai 2003).

34 Jérome Bel, Catalogue raisonné, 1994-2008, Coproduction : Les Laboratoires d'Aubervilliers, R. B./Jérôme Bel, 2007.

35 Entretien avec Jérôme Bel par Florian Gaité, « De la black box au white cube », Repères, cahier de danse, « Danse et/au musée », Vitry-sur-Seine, La Briqueterie, 2017, pp. 34-37.
} 
Figure 4 : $\quad$ Lynda Gaudreau, Out of Grace, Montréal, 2010. Vue de l'exposition, Galerie Leonard \& Bina Ellen et la Compagnie De Brune. Photo reproduite avec l'aimable autorisation de (C) Paul Smith.

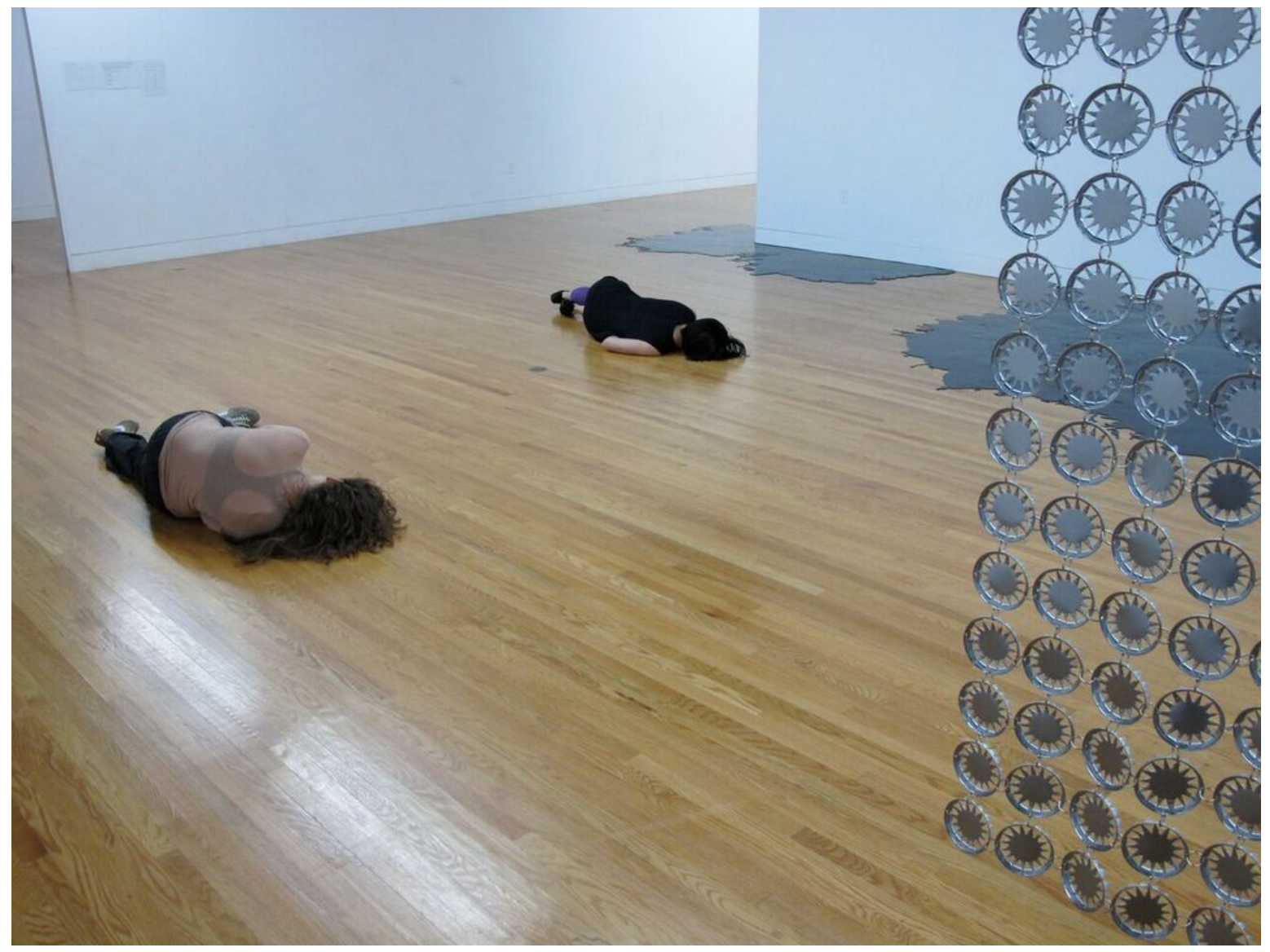

Pour les pionniers, le musée a toutefois pu offrir un terrain d'expérimentation. Merce Cunningham invente les Museum Events (le premier en 1964 au Musée des 20 Jahrhunderts, Vienne, Autriche). Plusieurs actions de courtes durées se produisent simultanément et permettent à la danse d'exister dans un espace non théâtral. Anna Halprin investit le Musée d'art moderne de San Francisco en 1967, à l'invitation du plasticien Bruce Conner. Peu après, elle participe à l'inauguration du Berkley Art Museum avec sa pièce Parades and changes. Son élève, Trisha Brown, présente Walking on the Wall au Whitney Museum of American Art à New York en 1971.

Leurs héritiers partagent-ils les aspirations de cette communauté artistique bouillonnante et très interdisciplinaire ? Paradoxalement, ces dernières années, la teneur conceptuelle de démarches qui ramènent la danse à ses conditions de possibilités minimales (lumière, musique, corps), se trouve à l'approche du musée, exposée à une forme de spectacularisation dont chacune des disciplines - danse et arts visuels - entendait pourtant se déprendre. S'il fallait s'en convaincre, la prolifération de séquences dansées qui ont fleuri les soirs de vernissages, en atteste (les arts visuels seraient-ils trop rébarbatifs qu'il leur faille le secours des arts vivants?).

Bien sûr, le musée a connu un précédent avec la performance par laquelle les arts visuels se sont ouverts au corps vivant, mais les enjeux et le contexte diffèrent désormais. 
L'engouement grandissant et l'inflation du «tout performatif » peut laisser dubitatif. Fervent défenseur de la performance à Québec, Richard Martel préfère d'ailleurs marquer la distinction par le terme de «Art-action ». Et de rappeler la filiation de la performance avec dada, puis l'action painting, l'actionnisme, le happening (etc.), davantage qu'avec une réflexion de nature chorégraphique. Serait-ce une pratique assagie et délestée de toute transgression dont l'institution est devenue si friande ? C'est la conclusion tirée par Claire Bishop qui constate que la danse performative a aujourd'hui largement perdu la charge critique et expérimentale de la performance des aînées, issue des arts visuels ${ }^{36}$.

Si l'écueil est réel, la relation de la danse et du musée ne s'y épuise pas. En revanche, comme la chorégraphe Lynda Gaudreau le souligne, dissocier la présence du corps de la notion de spectacle devient un enjeu. À cette fin, son dispositif Out of Grace (Galerie Leonard \& Bina Ellen, Montréal, 2010) propose une interaction permanente des plasticiens invités et de leurs œuvres avec les danseurs pendant les cinq semaines d'une « exposition qui commence en chorégraphie pour se terminer arts visuels ${ }^{37} \gg$. La forme ouverte qu'elle lui donne, non prédéfinie, questionne le formatage et le déterminisme culturel qui, régulant la manière de montrer un corps ou un objet, contraint habituellement la liberté du regardeur.

De part et d'autre, la dimension conjoncturelle n'est pas étrangère non plus à l'entrée de la danse au musée. Il n'est pas rare que des contraintes, en particulier budgétaires, puissent constituer le terreau d'une recherche artistique. Non sans ironie pour une discipline qui souffre de la précarité de son secteur, la danse serait une manne pour le musée. Réciproquement, elle trouve au musée un accueil pour des formes expérimentales que les théâtres peinent à accompagner.

Le passage d'un lieu à l'autre charrie un lot de considérations concrètes, qui ne manque pas d'accuser l'inadaptation du musée. L'intensité et la concentration risquent d'être malmenées par les circulations permanentes des visiteurs et d'entamer l'unité d'une proposition, sauf à en ramasser la forme dans l'instant pour pouvoir embrasser la pièce d'un regard. L'éclairage et l'acoustique ne répondent pas davantage aux attendus d'une salle de spectacle. D'aucuns se sont également élevés contre les conditions souvent éprouvantes auxquelles les danseurs sont soumis (l'amplitude horaire, l'absence de loges, de douches, la dureté des $\operatorname{sols}^{38}$ ).

\section{Black box, White cube, Grey box : des enjeux esthétiques et institutionnels croisés}

Et pourtant, le musée serait-il devenu un paradigme pour la danse ?

À peine directrice du Centre National de la Danse (Paris), Mathilde Monnier, ne le rebaptise-t-elle pas «Centre d'art de la danse », avant qu'elle n'accueille en son sein la

36 Claire Bishop, "The Perils and Possibilites of Dance in The Museum : Tate, MoMA, and Whitney ", dans Dance in the museum: Dance Research Journal, vol. 46, no. 3, Éditions Mark Franko et André Lepecki, Cambridge University Press, 2014. pp. 63-76.

37 Eloïse Guénard, « Entretien avec Lynda Gaudreau », 7 mars 2017, non publié, réalisé dans le cadre d'une résidence à LA CHAMBRE BLANCHE, en partenariat avec le MAC VAL - Musée d'Art contemporain du Val-de-Marne.

38 Le recueil d'entretiens Who cares? Dance in the Gallery \& Museums posait la question des bonnes pratiques à travers les témoignages de danseurs, curateurs, etc., Sarah Wookey, Londres, Siobhan Davies dance, 2015. 
manifestation «L'invitation aux musées ${ }^{39}$ » (2018) ? Lorsqu'il crée le Musée de la danse (Rennes, 2009), Boris Charmatz y voit un « un croisement contre-nature », « un paradoxe qui tire sa dynamique de ses propres contradictions », qui «voudrait bousculer l'idée que l'on se fait du musée, et l'idée que l'on se fait de la danse ! Mariage impossible entre deux mondes, il explore les tensions et les convergences entre arts plastiques et arts vivants, mémoire et création, collection et improvisations sauvages, œuvres mouvantes et gestes immobiles $^{40} »$. Citons encore, Dena Davida qui qualifie ses fonctions au sein de Tangente (acteur de majeur de la danse au Québec) de commissaire des arts vivants. L'appellation revendique en l'occurrence une dimension de recherche et d'expertise, qui entend rompre avec un «modèle d'affaire ${ }^{41}$ » encore dominant dans l'administration du spectacle vivant.

Figure 5 : $\quad$ Anne Teresa De Keersmaeker, Work/Travail/Arbeid, produit par Rosas \& WIELS, Centre Pompidou, réalisé avec le concours de l'Opéra de Paris, Paris, 2016. Photo reproduite avec l'aimable autorisation de (C) Anne van Aarschot.

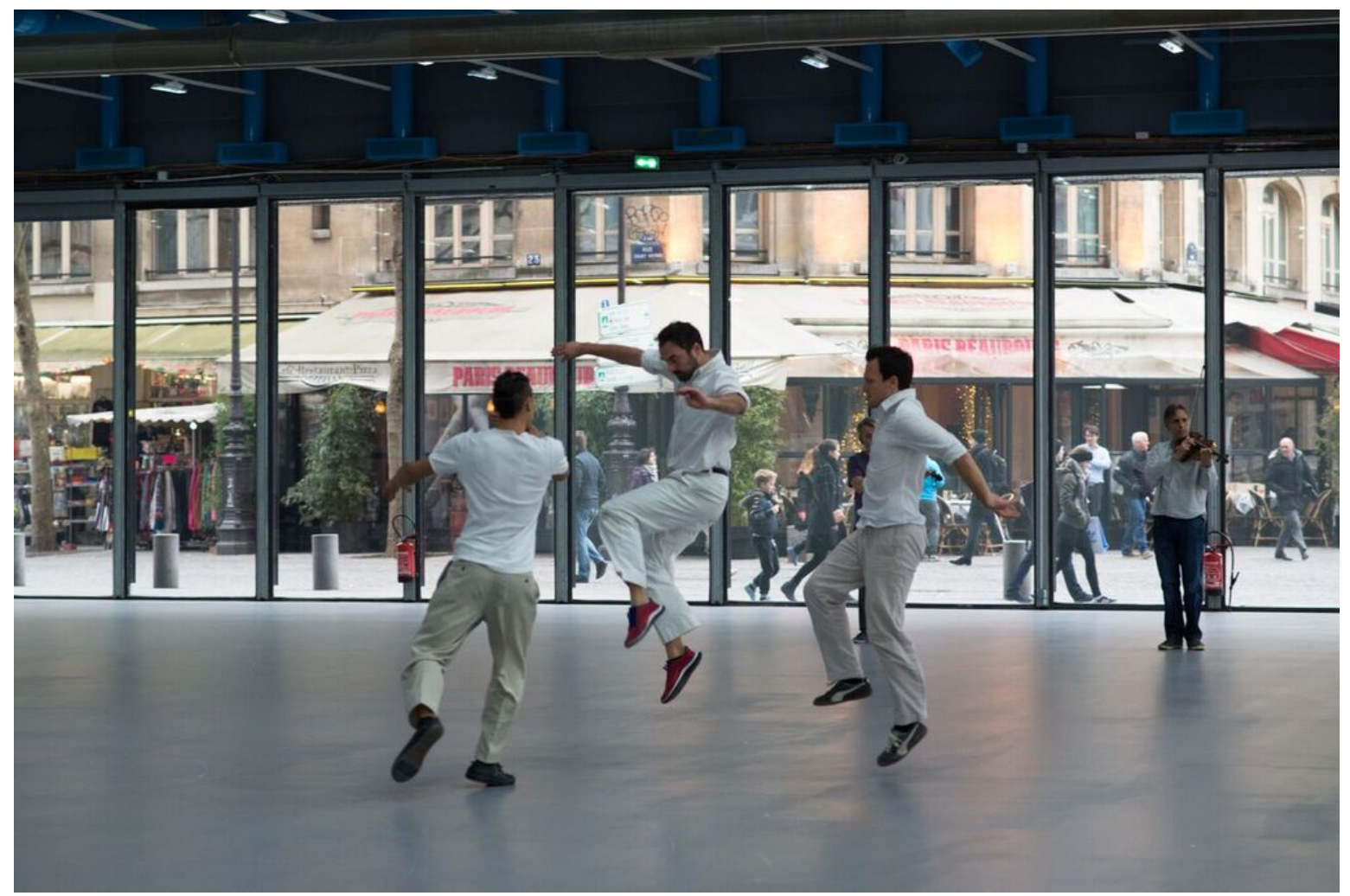

Ce mariage impossible, Laurence Louppe l'évoquait déjà dans sa délectable tirade sur la boîte noire et le White cube dans Dispositif 3.1 d'Alain Buffard (Centre Pompidou, 2001). Recroquevillée dans un carton d'emballage, elle déclarait : "C'est un retournement de

39 Extrait du dossier de presse : «Assumant plus que jamais le projet d'être un centre d'art pour la danse, le CN D invite six lieux d'art du monde entier à le transformer en un musée éphémère et vivant » (l'Institut d'Art de Chicago, le Musée national centre d'Art Reina-Sofía, Serralves - Musée d'art contemporain, le MAGASIN des horizons, le Musée de la danse, le Musée éphémère de la mode.)

40 Extrait du manifeste, site web du Musée de la danse : http://www.museedeladanse.org/fr/articles/musee-de-ladanse.html

41 Eloïse Guénard, « Entretien avec Dena Davida », 28 février 2017, non publié, réalisé dans le cadre d'une résidence à LA CHAMBRE BLANCHE, en partenariat avec le MAC VAL - Musée d'Art contemporain du Val-de-Marne. 
l'histoire de l'art que je suis en train de vivre et je suis vraiment heureuse car le cube blanc a toujours été mon idéal : être un sujet neutre dans un espace neutre qui fait des gestes neutres (...) ». Essoufflée, coiffée d'une perruque blonde, elle finissait par être rejetée du White cube de pacotille. Et pour cause ! «En l'état où je suis, il dit que je ne peux pas prétendre au statut de sujet neutre. Il me dit que, si je ne suis pas contente, je peux toujours me recycler dans l'esthétique relationnelle... ${ }^{42}{ }^{2}$. Parodie de la conception moderniste de l'espace muséal comme «White cube », tel que Brian O'Doherty ${ }^{43} 1$ 'a analysé, cette scène s'amuse d'une idéologie que le corps vient compromettre.

À rebours de l'idée d'autonomie et de neutralité, les «situations construites » de Tino Sehgal faites de séquences chorégraphiques et d'instructions orales transmises aux interprètes, ne produisent rien, sinon des relations sociales ${ }^{44}$. De ses pièces présentées dans de nombreuses institutions à travers le monde, rien ne perdure à l'expérience partagée dans l'instant. L'interdiction de prise de vue et de trace résiduelle prononcée par le chorégraphe s'étend à l'absence de tout document préalable, de catalogue et de cartel. Ultime déstabilisation des usages muséaux, les conditions d'acquisition et d'installation de l'œuvre sont énoncées sans aucune contractualisation écrite. Loin d'arracher un moment d'éternité au musée, la danse éprouve alors l'expérience du temps qui passe, avec pour corollaire la disparition. Elle ne désavoue pas en cela les arts visuels, devenus contemporains, pourraiton dire, par la réévaluation de leur prétention atemporelle.

La pérennité ne saurait-elle exister en danse, sinon dans un mouvement continu ou actualisé par l'instant présent ? Reprise de Vortex temporum (musique de Gérard Grisey), imaginé pour la scène, Work/travail/arbeid d'Anne-Teresa de Keersmaeker (2016, Centre Pompidou) réinvente l'espace-temps de la représentation pendant les neuf semaines d'exposition. Adoptant une forme cyclique, les mouvements de chaque danseur s'agrègent à la partition d'un des six musiciens de l'ensemble Ictus dans un tourbillon perpétuel. Le présent, commente la chorégraphe, «oscille perpétuellement entre souvenir et pressentiment, c'est un va-et-vient entre l'image résiduelle du passé et un désir d'avenir ${ }^{45}{ }$.

Les transferts de méthodes, de concepts et de pratiques entre danse et arts visuels au sein du musée entraînent l'inscription dans une temporalité et un espace réels, partagés par le public. Le musée, qui s'en était prémuni, est-il alors requalifié en Grey box ${ }^{46}$ qui, du temps de l'œuvre à l'œuvre dans le temps, repense l'historicité de la danse ?

42 Transcription de la captation vidéo du Dispositif 3.1 d'Alain Buffard.

43 Brian O'Doherty, White cube, l'espace de la galerie et son idéologie, Zurich, JRP/Ringier, co-éd. Paris, La maison rouge, 2008, Édition originale 1976-1986.

44 Présentées notamment au Walker Art Center (Minneapolis, 2008), au Musée Guggenheim (New York, 2010), à la Documenta (Kassel, 2012), à la Tate Modern (Londres, 2012), au MAC (Montréal, 2013), au Palais de Tokyo (Paris, 2016), à la Fondation Beyeler (Bâle, 2017).

45 Extrait du site web de la compagnie : https://www.rosas.be/fr/productions/386-vortex-temporum

46 L'expression est empruntée à Claire Bishop, «The perils and possibilites of dance in The Museum : Tate, MoMA, and Whitney »,op. cit.. 


\section{Références}

COPLAND, Mathieu (dir.). Chorégraphier l'exposition. Saint-Gall et Noisiel, Éditions Mathieu Copland, la Ferme du Buisson et la Kunst de Saint-Gall, Collection Hors série, 2013. 424 p.

O'DOHERTY, Brian. White cube, l'espace de la galerie et son idéologie. Zurich et Paris, JRP/Ringier et Maison Rouge, 2008 (Éd. originale 1976-1986). 208 p.

FRANKO, Mark et André Lepecki (dir.). «Dance in the museum » dans Congress on Research in Dance : Dance Research Journal, vol. 46, no. 3, Cambridge University Press, 2014. pp. 1-5.

« Danse ${ }^{\text {et/au }}$ musée », dans Repères, cahier de danse, no. 38-39, Vitry-sur-Seine, LaBriqueterie, mars 2017. pp. 5-9

POUILLAUDE, Frédéric. Le désoeuvrement chorégraphique, étude sur la notion d'œuvre en danse. Paris, Vrin, 2009. 274 p.

WOOKEY, Sarah. Who cares? Dance in the Gallery \& Museum. Londres, Siobhan Davies Dance, 2015. 161 p. 